\title{
Investigating the Dynamic Security of Power System to Detect System Stability or Instability by Using Neural Network
}

\author{
Reza Bagheri Ghahvachi \\ Electrical Department of Tehran Azad University, Tehran,Iran
}

\begin{abstract}
Modern power systems are very complex due to constant variations of the load. These systems are constantly exposed to internal and external disturbances that can cause system instability. The process of determining the stability of a system under turbulence is called security assessment. In other words, the security assessment of the power system is performed to determine the stability or instability of the system. The security assessment of the power system is a combination of static and dynamic security analysis. One of the ways to determine the dynamic security is to find the critical time to fix the fault. This time is a combination of functions with many variables, so its acquisition is relatively difficult. In addition, finding and evaluating the critical time of fault correction requires detailed and timely computations. Therefore, data classification can be used as the best option for assessing the security of a power system. Data classification, sampled data and computational time reduces security assessment. In this paper, three methods are used for classifying data. These methods include: least squares (correlation), Kohonen neural network and wavelet transform. The use of these methods eliminates the problems and issues that traditional methods have. If the classification of data is correct with the methods mentioned for input patterns and the critical times to correct the existing fault, then these methods can be used to determine the critical lines of the new input patterns without performing detailed calculations of transient stability.
\end{abstract}

Keywords: Power System - Neural Network - Dynamic Security - Critical Fault Time

DOI: $10.7176 / \mathrm{NCS} / 10-02$

Publication date:July $31^{\text {st }} 2019$

\section{Introduction}

The trend of increasing electric energy consumption and the need for reliable energy through the economic use of production resources has brought about changes in the network. One of the most important of these changes is the interconnection of regional networks, the interconnection of the power grid to each other, the use of serial capacitors in lines to increase transmission capacity, the use of high-pressure direct current lines and the use of voltage regulators. These changes have created new issues in all areas of network performance. And has led to the development of power system analysis techniques through the development of digital computing methods. And it should be noted that, unlike other laws and regulations in electrical engineering, the bases for analyzing power systems are based on hypotheses and methods that have gained years of experience.

Control and security is one of the most important issues that should be studied and studied in today's power networks based on the vision of a dynamic system and based on its results, the development of the future network and the improvement of the existing network, so that such a network can be in place at any moment, securely give consumers the energy they need.

One of the ways to determine the dynamic security is to find the critical time to fix the fault. This time is a combination of functions with many variables, so its acquisition is relatively difficult. The main purpose of this study is to obtain a method that can detect the transient stability of the critical network lines of each working pattern without any detailed calculations (finding the critical fault times), and also we can categorize the various work patterns of the network in terms of security. To diagnose system work patterns, in order to assess the dynamic security, three methods can be used.

One of the ways to determine the dynamic security is to find the critical time to fix the fault. This time is a combination of functions with many variables, so its acquisition is relatively difficult. Therefore, data classification can be used as the best option for assessing the security of a power system. The classification of data, sampled data, and computational time reduces security assessment. The main purpose of this study is to obtain a method that can detect the critical network lines of each working pattern without passing detailed computations of the transient stability (Finding critical time of solving fault), and also we can categorize various network work patterns in security.

\section{Literature review}

In the early 1950s, analog computers were used to investigate issues requiring a more detailed modeling of the synchronous machine, the acceleration system and the speed governor. Such simulations were suitable for the indepth study of the effect of equipment characteristics and not the overall behavior of the multi-machine system. In 1956, the first study of the stability of power system on digital computers was implemented. It soon became 
clear that these computers were superior to network analyzers for studying the network in terms of network size and dimension, and the possibility of more detailed modeling of the dynamic characteristics of the equipment. These were an appropriate means for studying the stability issues of interconnected systems that previously operated as separate systems. In the 1960s, most power systems in the United States and Canada became part of two major interconnected systems in the East and the other in the West. And in 1967 low-power communication lines were established between the East and West systems. Since the 1960s, much industry has focused on the sustainability of the system. Power systems were designed and exploited from the standpoint of criteria for transient stability. As a result, the main tool for sustainability research in design and operation is the transient stability program. So, over time, rapid fault equipment, fast response systems, serial capacitors and specific stability enhancement techniques have been developed. In recent years, there has been a lot of research and development that has helped to better understand the physical aspects of sustainability issues as well as the creation of the computational tools needed to better assess the system. [1]

A power system consisting of various elements such as synchronous machine, transmission line, load, power supply systems (boiler, ...), turbine (water, gas, steam), Automatic Voltage Regulator (AVR), smut transducer, systems of Responsive Power Provider (SVC) and so on. As a result, it can be said to be a dynamic system power system.

The main reason for the dynamism of most systems is that there are energy storage elements (inductors and capacitors in electrical circuits, masses and springs in mechanical systems, etc.). The presence of these elements causes the connection between the momentary input and output, for example, the capacitor in a circuit, is discharged by applying the voltage, charging and eliminating the voltage. Or, if we push the motorbike, then the mass of the automobile stores energy and then goes on, and when the power is cut off, it stores the stored energy and then stops. In a mass and spring system, if the material is drawn out and then released, it is exchanged between the mass and the energy of the spring. Kinetic energy is converted into potential energy and vice versa. And this causes mass fluctuations. In an orbiting circuit, consisting of an inductor and a capacitor, the inductor and the energy capacitor are exchanged and oscillations in the voltage and current are created.

The purpose of a power system is to provide the active power and the reactive power required by the consumer at the desired frequency and voltage. These four variables are monitored at each corner of the system, not only within a reasonable range, but also after the turbulence fluctuates rapidly and all more importantly, the fluctuations are stable.

In terms of dynamic studies, frequency control and voltage control are the most important dynamical problems of power systems. Although issues of frequency and voltage control are not independent, they are often considered independently. In addition, in studying frequency fluctuations, the angular load fluctuations usually follow the same fluctuations with a phase difference. [2]

Edison founded the first generation and distribution system in 1882 in New York City. The basis of this system was direct voltage and direct current for a total of 59 customers in a 1.5 kilometer radius of light. Since then, the power generation and distribution industry has undergone many changes and changes. In the first system of production and distribution of electricity, dynamic and control studies may not have been considered and considered by the designer, but nowadays it is not possible to exploit the electrical system without dynamic studies.

After the advent of the electric motor (1884) and the transformer (1886), the generation and distribution of alternating electric power were considered. Because in this system, by increasing the voltage with the transformer on the side of manufacture and lowering it on the side of consumption, it can reduce the loss of the transmission line and can withstand the voltage drop in the transmission line, but because the storage elements energy is responsive to variations, and alternating current is constantly changing, dynamic studies in alternating current systems are more important than direct electricity.

Since power consumption varies in different regions and at different times. For optimal utilization of investment in the production sector, it is advisable to connect electricity generation and distribution networks in areas where the amount of demand requested is greater than the capacity produced or vice versa, an area can service another area.

Although the connection of small systems of production and distribution to one another, makes the system more stable against small disturbances, but if it is a serious problem for a system, it also affects other systems. For a better understanding of the subject, consider those who move down the slope down the slope. If each one acts independently, the smallest obstacle can not cause each of them to fall, but if these people go hand in hand this does not happen, and if one creates a big obstacle for one person, it will not only make it difficult for that person, but also balance the balance of other people. However, for reasons of economy, the network the modern production and distribution of electricity are highly interconnected, and not only the different regions of one country, but the networks of different countries and, ultimately, the network Production and distribution of different continents are connected. Although these connections have been made according to economic requirements, however, dynamic studies of power systems have become more and more necessary. 
Modern power systems have a lot of characteristics, such as production in higher capacity units and far away from the place of production and place of use (prolongation of power lines), which can be done for economic reasons, but on sustainability the system has a negative effect. Another characteristic of modern electricity production, which requires dynamic studies, is that for the maximum use of investments in the production of today's systems, the production in the electricity industry is usually at its highest capacity, this system stability issue Power low.

\section{Dynamic security assessment of the power system using the neural network}

The excessive development of the energy market has increased the use of power systems near the security margin. This has led to complex methods for identifying the vulnerability margin. The multidimensional nature of power systems creates problems. In these decades, dynamic security assessment methods have been developed based on time domain simulation and direct methods such as energy function assessment. Despite the advances in computing, these methods, although better for diagnosing security situations, require time calculations for accurate and reliable computing of security indicators or security margins. This is due to the complex and extensive structure of the functioning of modern power systems.

Over time, various kinds of neglect and partnerships in the energy structure make power systems more closely exploited by the security margin. Economic system approaches the security or margin of vulnerability, resulting in a total the system security margin decreases. Under this scenario, the operator should be fully aware of the security margin. If the calculations are too high, the previous methods are not responsive, since the margin is a dynamic level change, and in recent years, different methods and techniques have been used for the dynamic process of dynamic security evaluation, combined with new challenges, while the accuracy of the calculation is maintained.

Smart system technology has made good applications for the evaluation of dynamic security. Examples include neural networks, expert systems, and so on. When operating modes depend on a stable transition or security margin, neural networks are very much considered and different neural networks are used to identify the security margin.

In articles $[9,10,11]$, various systems are used to determine the power system security margin. Therefore, with the security margin of the operator, the power system can keep the system from harm. In all the articles studied, the classification method for the evaluation of dynamic security has not been used and it can be said that the method used in this project has been investigated for the first time. One of the advantages of this method is the speed of dynamic security assessment, so that in case of confusion, the critical lines of the system can be detected within a few seconds and the operator can take preventive measures to prevent the network instability.

The IEEE 39-bus Standard Network

The IEEE 39-bus standard power system is shown in Fig. (1). Bus 31, whose voltage is determined $\mathrm{V}_{31}=0.9820$ $<0 \mathrm{pu}$, is selected as the reference point.

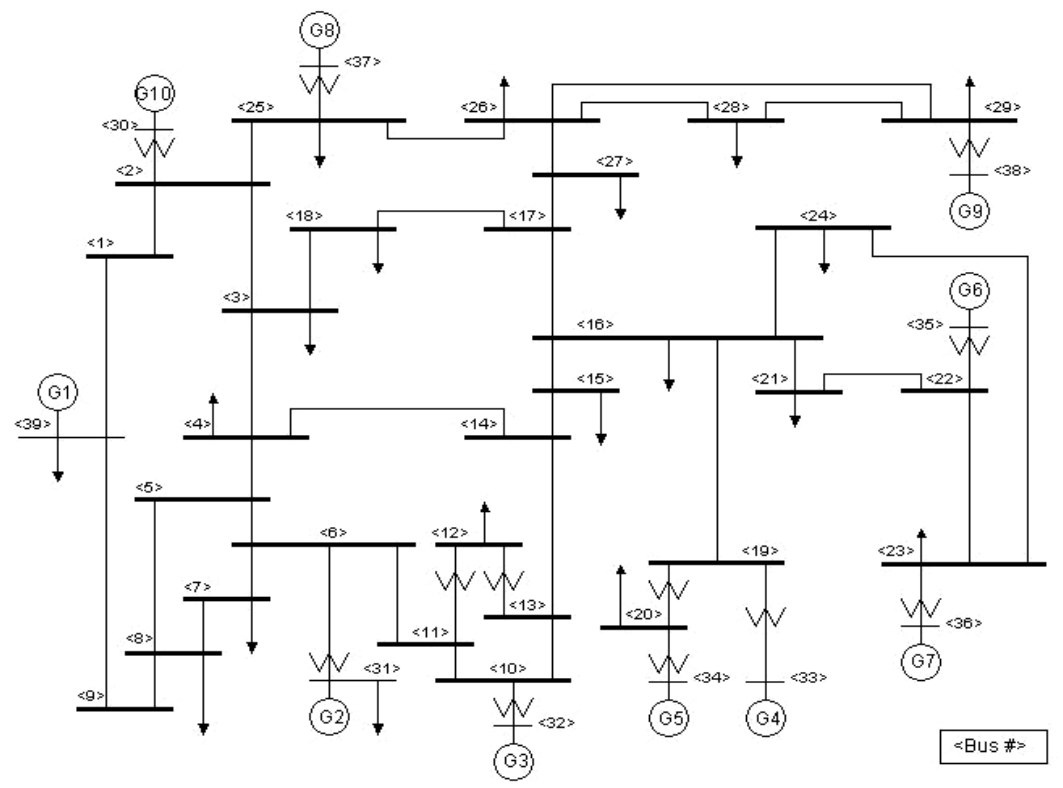

Figure (1): The IEEE 39-bus standard system 
For any power system operation, the system's stable operating conditions in the Pre-Fault state 1 can be demonstrated by the work pattern ( $\mathrm{P} \Phi V)$, which is proportional to the number of shins and network lines, and includes all variables of system operation at the point of operation. And the frame of each system pattern can be used to calculate and determine the corresponding behavioral pattern (CCT); in Figure 2-5, the combination of both patterns is shown.

\begin{tabular}{|c|c|c|c|c|c|c|c|c|}
\hline & $\mathrm{N}_{\mathrm{B}}$ & $\mathrm{N}_{\mathrm{B}}$ & $\mathrm{N}_{\mathrm{B}}$ & $\mathrm{N}_{\mathrm{B}}$ & $\mathrm{N}_{\mathrm{L}}$ & $\mathrm{N}_{\mathrm{L}}$ & $\mathrm{N}_{\mathrm{B}}$ & $\mathrm{N}_{\mathrm{B}}$ \\
\hline $\mathrm{P} \Phi V=$ & $\mathrm{P}_{\mathrm{G}}$ & $\Phi_{G}$ & $\mathrm{P}_{\text {Load }}$ & ${ }_{\text {Load }} \Phi$ & $\mathrm{P}_{\text {Line }}$ & $\Phi_{\text {Line }}$ & $V_{B u s}$ & $\theta_{\text {Bus }}$ \\
\hline
\end{tabular}

\begin{tabular}{r|r|r|r|rrr|r|r|}
$\mathrm{CCT}=$ & $\mathrm{CCT}_{1}$ & $\mathrm{CCT}_{2}$ & $\mathrm{CCT}_{3}$ & $\ldots \ldots \ldots$ & $\ldots \ldots \ldots$ & $\ldots \ldots \ldots$ & $\ldots \ldots \ldots$ & $\mathrm{CCT}_{\mathrm{NL}}$ \\
\hline
\end{tabular}

Figure (2-5): Combination type of working and behavior patterns

$\mathrm{N}_{\mathrm{B}}$ : Number of bus.

$\mathrm{N}_{\mathrm{L}}$ : Number of lines.

The behavioral pattern of the system represents the CCT corresponding to each of the network lines, so for the work pattern number $\mathrm{K}$, means $\mathrm{P} \Phi V_{K}$ respectively, there will be a behavioral pattern of the number $\mathrm{K}$, namely, $\mathrm{CCT}_{\mathrm{K}}$, which is extracted through the transient stability calculation, in fact we know that:

$$
\mathrm{CCT}_{\mathrm{K}}=\mathrm{f}(\mathrm{)}) \mathrm{P} \Phi V_{K}
$$

To determine the correlation between work patterns, the deviation index based on the least squares criterion can be used as follows:

$$
\delta_{P \Phi V j k}=\frac{1}{N}\left[\sum_{i=0}^{N}\left[x_{j i}-x_{k i}\right]^{2}\right]^{1 / 2}
$$

$\mathrm{Xj}$ : Elements of the working pattern $\mathrm{j}$, which are characterized by index $\mathrm{i}$.

$\mathrm{Xji}$ : The i-th element of j's work pattern.

Xki: The i-th element of the k-template.

$\mathrm{N}$ : The number of elements of each of the working patterns $\mathrm{j} k$,

$\delta_{\mathrm{P} \Phi V j k}$ : Deviation of working patterns $\mathrm{k}$, j based on the least squares criterion.

To calculate and determine the correlation between behavioral patterns corresponding to work patterns, the deviation index based on the least squares criterion can be used as follows:

$\delta_{C C T j k}=\frac{1}{N L}\left[\sum_{i=0}^{N L}\left[C C T_{j i}-C C T_{k i}\right]^{2}\right]^{1 / 2}$

So that:

CCTji: The CCT corresponding to the $\mathrm{i}$-th line in the model number $\mathrm{j}$.

CCTki: The CCT corresponding to the i-th line in the k-model.

$\delta_{\text {CCTjk }}$.

Deviation of behavioral patterns $\mathrm{k}, \mathrm{j}$ based on the least squares criterion.

NL: The number of elements of each behavioral pattern $\mathrm{j} k$,

\section{Neural Network Functional Pluralization of Security Analyst}

To demonstrate the performance of the neural network, the security analyst in the security classification of the system seeks to match the classification of the exploitation pattern 1517 and is shown in Fig. 2. With regard to seeking weight matching of 68 , it can be concluded that seeking a good weight matching between work and behavioral patterns.

Therefore, we have been able to classify proper behavioral and behavioral models with the Kohonen Neural Network. We conclude that using this neural network, we can identify the critical lines with the potential for transient instability risk for the network, based on the working pattern of each operating condition. 


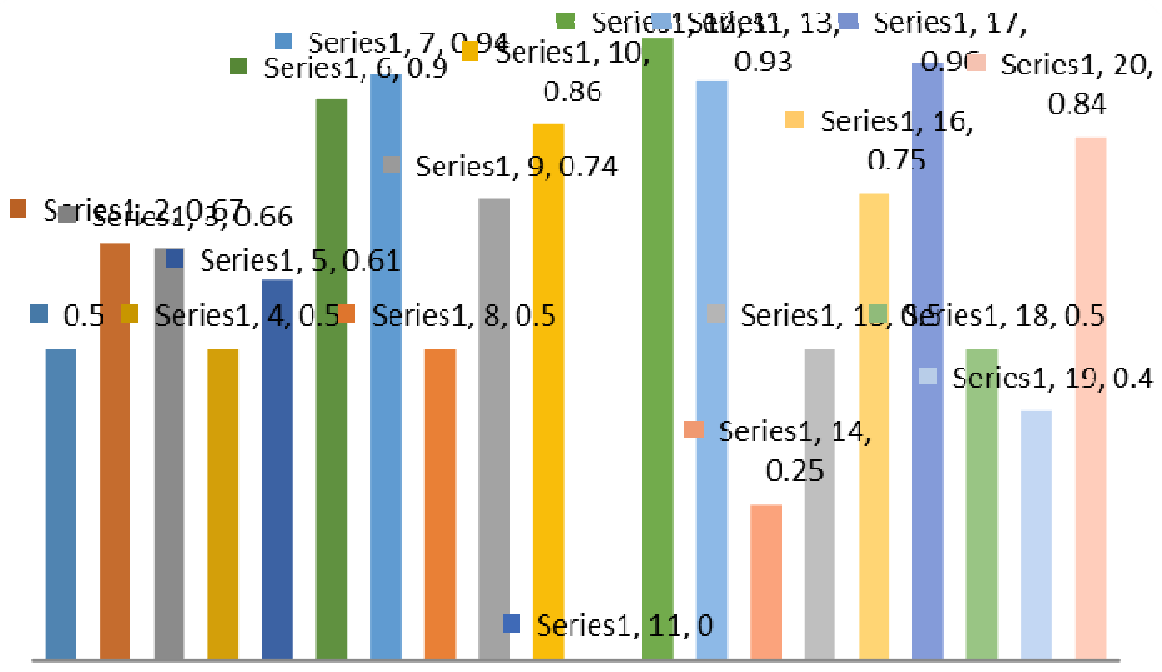

Figure (2): Adaptations percentage of different classes in neural network testing

\section{Wavelet analysis}

Wavelet is derived from the French word Ondelette, meaning a small wave. Over time, it has become a powerful mathematical tool for signal analysis. The use of Wavelet does not deny the Fourier method, but the continuation of Joseph Fourier in the nineteenth century and is known as a complement to Fourier transformation.

Wavelet is an oscillating function with a mean zero that is rapidly zero (the beginning and the end of the signal is zero). And unlike the shape of sinusoidal waves that are not limited (from $-\infty$ to $+\infty$ ), they are smooth and predictable, the wavelets are irregular and asymmetric. It can be said that Wavelet is a transient signal, and WT is very effective for analyzing transient states. Figure (3)
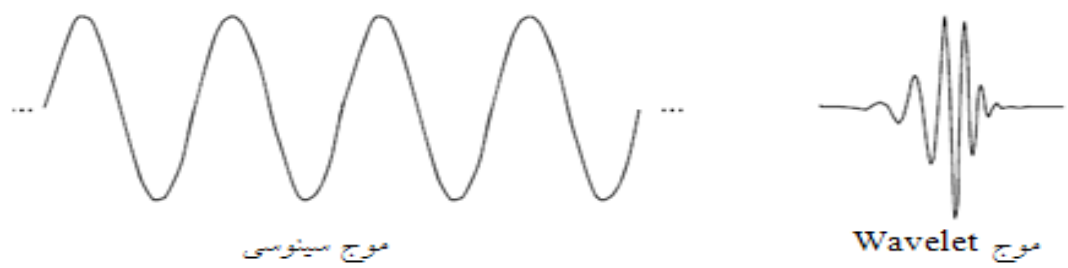

Figure (3): Comparison of Sine Waveforms and Wavelet

Wavelet analysis is the same as the STFT analysis with variable ranges. In this analysis, long distances are used for low-frequency information and short distances for high-frequency data. In the simplest way, if we consider a large range of signals, we will see the major indicators (fundamental changes) of the signal and, similarly, by considering the small range of the signal, we will see the fine indexes (small changes) of the signal.

One of the important benefits of Wavelet analysis is the ability to perform local analysis (regional analysis of a large signal). Local analysis provides us with unique information that other techniques cannot do, such as breaking points, signal discontinuities, and so on. For example, disturbances in a sinusoidal function may not be detectable due to a small amount. With the Fourier transform and the Fourier coefficients of this function, there is nothing significant to note while WT and Wavelet coefficients are clearly identifiable precisely the disturbance in the time domain.

In Wavelet analysis, unlike Fourier analysis, many functions are used as the main function, and this frequency is quite distinct with different Wavelet names.

The mathematical formula in the Fourier analysis process is represented as Fourier transform:

$$
F(w)=\int_{+\infty}^{-\infty} f(t) \cdot e^{-j w t} d t
$$

\footnotetext{
${ }^{1}$ Wavelet Transformer
} 
Fourier coefficients $\mathrm{F}(\mathrm{w})$ are the sum of the product of the function $\mathrm{f}(\mathrm{t})$ and the exponential function ( $)$, which is the product of these coefficients and sinusoidal functions with different frequencies w, constitute the sinusoidal components of the main signal. The sinusoidal components are graphically arranged in Figure 4 is depicted.

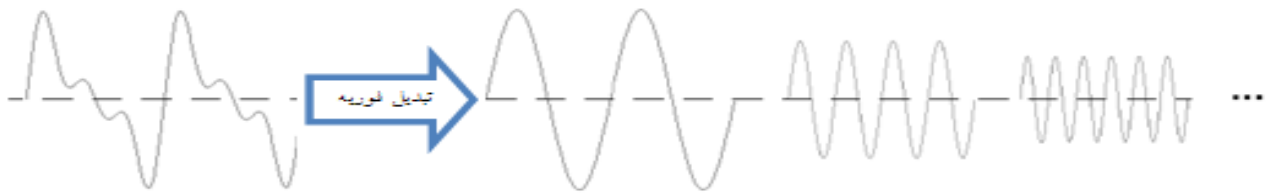

Figure 4: Main signal along with sinusoidal components with different frequencies

Similarly, CWT is the sum of product of the function $f(t)$ and the wavelet function depends on the size $\Psi($ scal, position)

(expansion and contraction of the signal) and the movement, it means

$$
C(\text { scal }, \text { position })=\int_{+\infty}^{-\infty} f(t) . \Psi(\text { scal }, \text { position }) d t
$$

The resulting wavelet transform coefficients are a function of size and displacement, in fact, these coefficients can be called the index of similarity between the main signals and the wavelet. And the product of any coefficient with proper Wavelet in terms of size and displacement, provides wavelets of the original signal, which is graphically depicted in Fig. 5 .

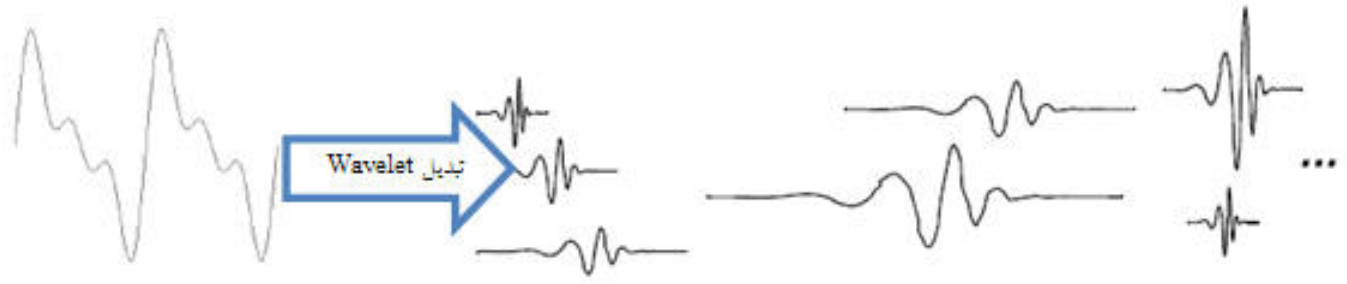

Figure (5): Main signal and different alternating waves

First of all, we mean the size and displacement in the Wavelet function. The size in Wavelet simply means the expansion and contraction (compression) of the signal, and for a better understanding, we define a coefficient called the coefficient of size (a). When such a coefficient is used in the wavelet, it is observed that the lowest coefficient with the highest density is associated with. Figure (6) clearly shows the user the size factor.

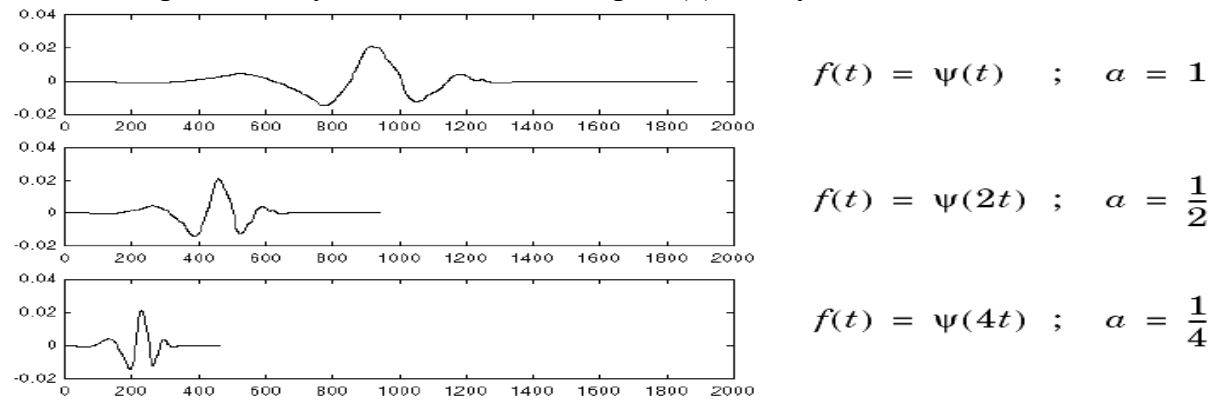

Figure (6): Applying the size coefficient in the Wavelet

But moving to a point in the wavelet is simply delayed or accelerated at the start points, and from the mathematical point of delay the function $\mathrm{f}(\mathrm{t})$ is expressed as $\mathrm{f}(\mathrm{t}-\mathrm{k})$. Fig. 7 

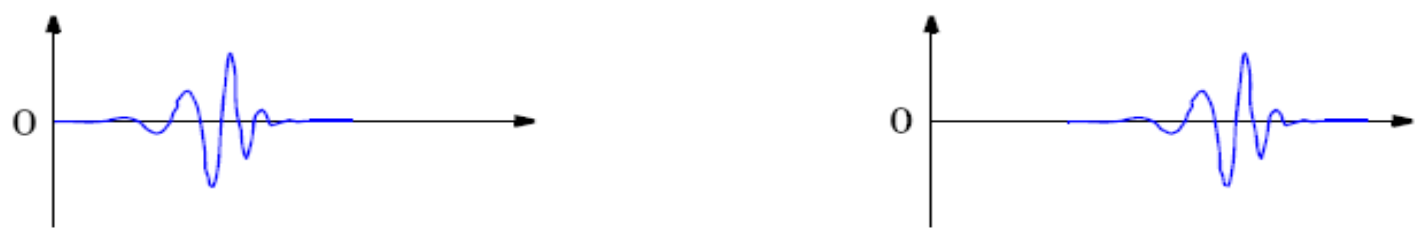

Figure 7: Wavelet signal with displacement

The most important part of many signals are low frequencies, and with these frequencies, signals can be detected. On the other hand, the high frequencies express the exact points of the signal.

Since WT plays an important role in the size and frequency, its relationship with the filtration stage must be determined, this relationship is as follows:

Large sizes $\Rightarrow$ Low frequency components of signal $\Rightarrow$ Assessments

Small sizes $\Rightarrow$ High frequency components of signal $\Rightarrow$ Details

In order to reduce the additional information, a concept called the sampling reduction is introduced, and this is really a simple tool for reducing the samples, so with this filter output, the passage and downstream pass filter are called component coefficients and estimation coefficients, respectively. It should be noted that this creates unevenness in the components of the signal. The analysis process continues with continuous analysis of estimates in each step, so the signal is decomposed into smaller components. This continuous decomposition is known as the Wavelet Decomposition Tree. Regarding the decomposition tree, it can be seen that WT outputs are coefficients of detail and estimates, but in practice WT outputs include components (D) and approximation (A) that are obtained using the coefficients of detail and estimation.

\section{Conclusion}

One of the ways to determine the dynamic security is to find the critical time to fix the fault. This time is a combination of functions with many variables, so its acquisition is relatively difficult. In addition, finding and evaluating the critical time of fault correction requires detailed and timely computations. Therefore, data classification can be used as the best option for assessing the dynamic security of a power system. Data classification, sampled data, and computational time reduces security assessment.

\section{Suggestions}

Considering the importance of determining the dynamic security of real power systems by advanced methods, including the method presented in this thesis, it can be obtained online in a critical time to correct the fault of the real power system and prevent preventive measures through probable instabilities.

\section{References}

1- Prof. Pourbarahr Shankar Condor, 1997, Stability and Control of Power Systems Vol. 1 \& 2, Tarbiat Modares University Press, 1 pp., 47 p., 21 p., 28 p., 34 p., 1021 p., 1030 p.

2- Dr. Mehdi Karari, 2003, Dynamics and Control of Power Systems, Amir Kabir University of Technology, 2 pp., 5 pp., 9 p., 263 p., 351 p., 436 p.

3- J De. Gelver, 2001, Power Systems Research and Design, Imam Reza University Press, 599 p.

4. Professor M. Anata Pei, 2003, Dynamics and Power Systems Sustainability, Gol Aftab Publishing, $348 \mathrm{pp}$

5th Machovsky, c. Bialek, 2005, Dynamics and Stability of Power Systems, Publication of Iran University of Science and Technology, $276 \mathrm{p}$.

6- Hadi Saadat, 2003, Investigating Voltage Systems (2), Iran University of Science and Technology

7- R Bill and T. Jackson, 2001, Introduction to Neural Networks, Sharif University Press, 96 p., 100 p., 102 p., $106 \mathrm{p}$.

8- Phyllit Piktan, 2004, Neural Networks, Iran University of Science and Technology, $198 \mathrm{p}$

9- I. N. Kassabalidis, M.A. El-Sharkawi, R.J.Marks; Dynamic Security Border Identification using Enhanced Particle Swarm Optimization . 2002

10- I. N. Kassabalidis, M.A. El-Sharkawi, R.J.Marks; Border Identification For Power System Security Assessment using Neural Network Inversion.

11- A. Sittithumwat, K. Tomsovic ; Dynamic Security Margin Estimation with Preventive Control using Artificial Neural Networks .2004

12 - A.J.Germond, D.Niebur ; Application of Kohonen Maps to Security Analysis .

13- T. Cornu , P.Ienne, D.Niebur ; A Systolic Accelerator For Power System Security Assessment.

14- H. Kim ; Evaluation of Power System Security and Development of Transmission Pricing Method .2003

15- T.Senjyu , Y.Tamaki ; Next Day Load Curve Forecasting using Self Organizing Map .2000 
16- J.F.Maqueen , A.O.Ekwue ; Application of Kohonen Self Organizing Neural Network to Static Security Assessment 1995

17- Neural Network Toolbox .

18 - A.W.Galli ; Wavelet Analysis for Power System Transients .

19- Wavelet Toolbox .

20- A.W.Galli , G.T.Heydt , P.F. ribeiro ; Exploring the Power of Wavelet Analysis .1996

21- A.M.Gaouda , M.R.Sultan ; Power Quality Detection and Classification using Wavelet Multiresolution Signal Decomposition. 1999

22- J.W.Resende , M.L.R.Chaves ; Identification of Power Quality Disturbances using the MATLAB Wavelet Transform Toolbox .

23- H.N.Rojas ,R.C.Fernandes ; An Overview of Wavelet Transforms Application in Power Systems . 2002 